IASSNS-HEP-99/85

September, 1999

\title{
Derivation of the Lindblad Generator Structure by use of the Itô Stochastic Calculus
}

Stephen L. Adler

\author{
Institute for Advanced Study
}

Princeton, NJ 08540

\author{
Send correspondence to: \\ Stephen L. Adler \\ Institute for Advanced Study \\ Olden Lane, Princeton, NJ 08540 \\ Phone 609-734-8051; FAX 609-924-8399; email adler@ias.edu
}




\begin{abstract}
We use the Itô stochastic calculus to give a simple derivation of the Lindblad form for the generator of a completely positive density matrix evolution, by specialization from the corresponding global form for a completely positive map. As a by-product, we obtain a generalized generator for a completely positive stochastic density matrix evolution.
\end{abstract}


Completely positive maps, and dynamical semigroups constructed from them, play an important role in the theory of quantum dissipative systems [1]. In the global case, the general form of a completely positive map $X \rightarrow T(X)$ has the simple and intuitive structure $[2]$

$$
T(X)=\sum_{n \in N} A_{n} X A_{n}^{\dagger}
$$

with the $A_{n}$ operators indexed by $n$. In the infinitesimal case, the most general generator of a completely positive density matrix evolution has the so-called "Lindblad form" [3]

$$
\mathcal{L} \rho=-i[H, \rho]+\sum_{n \in N}\left[v_{n} \rho v_{n}^{\dagger}-\frac{1}{2} \rho v_{n}^{\dagger} v_{n}-\frac{1}{2} v_{n}^{\dagger} v_{n} \rho\right],
$$

with $H$ self-adjoint. Although one normally expects a simple and evident correspondence between the global and infinitesimal forms of a transformation, the original proofs of Eq. (2) in Refs. [3] follow a less direct route. Recently, Peres [4] has made the interesting remark that the connection between Eq. (1) and the $v_{n} \rho v_{n}^{\dagger}$ term in Eq. (2) can be heuristically understood by identifying $v_{n}$ with a rapidly fluctuating part of $A_{n}$, of magnitude $(d t)^{\frac{1}{2}}$. Much the same physical idea is exploited in the book of Parthasarathy [5], to give a derivation of Eq. (2) using stochastic calculus methods. Our aim in this note is to sharpen Peres' observation by using the Itô stochastic calculus to obtain Eq. (2) directly as an infinitesimal specialization of the global transformation of Eq. (1). As a by-product, we will obtain a generalized form for the generator of an infinitesimal completely positive stochastic density matrix evolution.

We start by specializing Eq. (1) to operators $A_{n}$ of the form

$$
\begin{aligned}
& A_{n}=d_{n}+u_{n} d t+v_{n} d W_{t}^{n}, \\
& A_{n}^{\dagger}=d_{n}+u_{n}^{\dagger} d t+v_{n}^{\dagger} d W_{t}^{n} .
\end{aligned}
$$

Here the $d_{n}$ are positive real numbers; no extra generality is achieved by taking the $d_{n}$ 's as complex, since by redefining the $u_{n}, v_{n}$ a phase in $d_{n}$ can be transformed into an overall 
c-number phase factor in $A_{n}$, which does not contribute to Eq. (1). The $d W_{t}^{n}$ are Itô stochastic differentials [6] which obey the algebra

$$
d W_{t}^{m} d W_{t}^{n}=c^{m n} d t, \quad d W_{t}^{m} d t=0
$$

with $c$ a real symmetric and positive semidefinite covariance matrix. By appropriately normalizing the operators $v_{n}$, the diagonal matrix elements of $c$ can always be made equal to unity, so that we have

$$
c^{n n}=1 \text {, all } n \text {. }
$$

The definition of Eqs. (4) and (5) includes as special cases that in which the Itô differentials are all the same, $d W_{t}^{n}=d W_{t}$, all $n$ (for which $c^{m n}=1$, all $m, n$ ), and that in which the Itô differentials $d W_{t}^{n}$ are all independent( for which $c^{m n}=\delta^{m n}$ ).

Consider now the completely positive density matrix transformation

$$
\rho \rightarrow \rho+d \rho=T(\rho)
$$

Substituting Eq. (3) into Eqs. (1) and (6) and using Eq. (4), we get

$$
\begin{aligned}
\rho+d \rho & =\sum_{n \in N}\left(d_{n}+u_{n} d t+v_{n} d W_{t}^{n}\right) \rho\left(d_{n}+u_{n}^{\dagger} d t+v_{n}^{\dagger} d W_{t}^{n}\right) \\
& =\sum_{n \in N} d_{n}^{2} \rho+\sum_{n \in N} d_{n}\left(v_{n} \rho+\rho v_{n}^{\dagger}\right) d W_{t}^{n}+\left(\rho U^{\dagger}+U \rho+\sum_{n \in N} v_{n} \rho v_{n}^{\dagger}\right) d t
\end{aligned}
$$

with

$$
U=\sum_{n \in N} d_{n} u_{n}
$$

Equating the coefficients of $\rho$ on the left and right hand sides of Eq. (7a) gives the condition

$$
\sum_{n \in N} d_{n}^{2}=1
$$


while for the change in $\rho$ we get

$$
d \rho=\sum_{n \in N} d_{n}\left(v_{n} \rho+\rho v_{n}^{\dagger}\right) d W_{t}^{n}+\left(\rho U^{\dagger}+U \rho+\sum_{n \in N} v_{n} \rho v_{n}^{\dagger}\right) d t
$$

Let us now take the expectation value of Eq. (8) with respect to the stochastic process; since the operators $u_{n}, v_{n}$ have no dependence on the Itô stochastic differentials, and since $E\left[\rho d W_{t}^{n}\right]=0$ in the Itô calculus [6], we get simply

$$
d E[\rho]=E[d \rho]=\left(E[\rho] U^{\dagger}+U E[\rho]+\sum_{n \in N} v_{n} E[\rho] v_{n}^{\dagger}\right) d t
$$

Now let us impose the condition that the density matrix must always have trace unity, which implies that $\operatorname{Tr} d \rho=\operatorname{Tr} E[d \rho]=0$. From Eq. (9), we get by cyclic permutation under the trace

$$
0=\operatorname{Tr} E[\rho]\left[U+U^{\dagger}+\sum_{n \in N} v_{n}^{\dagger} v_{n}\right]
$$

which can hold for general $E[\rho]$ only if the coefficient operator is zero, which implies that

$$
U+U^{\dagger}=-\sum_{n \in N} v_{n}^{\dagger} v_{n}
$$

Thus the condition that the infinitesimal transformation preserve the trace of $\rho$ determines the self-adjoint part of $U$, while the anti-self-adjoint part can be an arbitrary operator $i H$, with $H$ self-adjoint, so that $U$ has the form

$$
U=-i H-\frac{1}{2} \sum_{n \in N} v_{n}^{\dagger} v_{n}
$$

Substituting Eq. (11) into Eq. (9) then gives for the completely positive, trace preserving infinitesimal deterministic evolution of $E[\rho]$ the expression

$$
\frac{d E[\rho]}{d t}=-i[H, E[\rho]]+\sum_{n \in N}\left[v_{n} E[\rho] v_{n}^{\dagger}-\frac{1}{2} v_{n}^{\dagger} v_{n} E[\rho]-\frac{1}{2} E[\rho] v_{n}^{\dagger} v_{n}\right]
$$


which is the Lindblad form.

Returning now to the stochastic evolution of Eq. (8), substituting the above results and the condition $\operatorname{Tr} d \rho=0$, we get the additional condition

$$
\operatorname{Tr} \rho \sum_{n \in N} d_{n}\left(v_{n}+v_{n}^{\dagger}\right) d W_{t}^{n}=0
$$

which can hold for general $\rho$ only if the operator coefficient vanishes, so that

$$
\sum_{n \in N} d_{n}\left(v_{n}+v_{n}^{\dagger}\right) d W_{t}^{n}=0
$$

Multiplying by $d W_{t}^{m}$ we get the equations

$$
\sum_{n \in N} d_{n}\left(v_{n}+v_{n}^{\dagger}\right) c^{n m}=0, \quad \text { all } m \in N
$$

The condition of Eq. (13c) can be rewritten by noting that since $c^{n m}$ is real symmetric and nonnegative, it is diagonalized by an orthogonal matrix $O^{m r}$ to yield nonnegative eigenvalues $c^{r}$,

$$
\sum_{m \in N} c^{n m} O^{m r}=O^{n r} c^{r}
$$

with no sum over $r$ on the right. Thus Eq. (13c) is equivalent to

$$
\sum_{n \in N} d_{n}\left(v_{n}+v_{n}^{\dagger}\right) O^{n r} c^{r}=0, \text { all } r \in N
$$

For those values of $r$ for which $c^{r}>0$, we can factor out $c^{r}$ to give the restriction

$$
\sum_{n \in N} d_{n}\left(v_{n}+v_{n}^{\dagger}\right) O^{n r}=0, \text { all } r \text { with } c^{r}>0
$$

while for those $r$ for which $c^{r}=0$, there is no restriction. Defining diagonalized Itô differentials $d Z_{t}^{r}$ by

$$
d Z_{t}^{r}=\sum_{n \in N} d W_{t}^{n} O^{n r}
$$


with the inversion

$$
d W_{t}^{n}=\sum_{r \in N} O^{n r} d Z_{t}^{r}
$$

we find that

$$
d Z_{t}^{q} d Z_{t}^{r}=\sum_{n, m \in N} d W_{t}^{n} d W_{t}^{m} O^{n q} O^{m r}=\sum_{n, m \in N} c^{n m} O^{n q} O^{m r} d t=\delta^{q r} c^{r} d t
$$

and that the restriction of Eq. (13b) takes the form

$$
\sum_{n, r \in N} d_{n}\left(v_{n}+v_{n}^{\dagger}\right) O^{n r} d Z_{t}^{r}=0
$$

Since Eq. (15c) implies that for those $r$ for which $c^{r}=0$ the corresponding $d Z_{t}^{r}$ is idempotent, and hence vanishes, the reduced set of restrictions given in Eq. (14c) suffices to guarantee the vanishing of Eq. (15d) and hence the satisfaction of the original condition of Eq. (13b). We conclude that the completely positive, trace preserving stochastic evolution of $\rho$ corresponding to our construction of Eq. (12) is generated by

$$
d \rho=\sum_{n \in N} d_{n}\left(v_{n} \rho+\rho v_{n}^{\dagger}\right) d W_{t}^{n}+\left[-i[H, \rho]+\sum_{n \in N}\left[v_{n} \rho v_{n}^{\dagger}-\frac{1}{2} v_{n}^{\dagger} v_{n} \rho-\frac{1}{2} \rho v_{n}^{\dagger} v_{n}\right]\right] d t
$$

subject to the restrictions on the positive real numbers $d_{n}$ and the operators $v_{n}$ of Eqs. (7c) and $(14 \mathrm{c})$.

The simplest case of Eq. (16) is that in which the sum over the index set $N$ contains only one term, so that Eq. (7c) implies $d_{1}=1$ and Eq. (5) completely determines the covariance matrix to be $c^{11}=1$. Dropping the superfluous index $n$, we get

$$
d \rho=\left(v \rho+\rho v^{\dagger}\right) d W_{t}+\left[-i[H, \rho]+v \rho v^{\dagger}-\frac{1}{2} v^{\dagger} v \rho-\frac{1}{2} \rho v^{\dagger} v\right] d t
$$

with the restriction of Eq. (14c) simplifying to

$$
v+v^{\dagger}=0
$$


which implies that

$$
v=-i K \quad,
$$

with $K$ self adjoint. Substituting Eq. (18b) back into Eq. (17), we get

$$
\begin{aligned}
d \rho & =-i[K, \rho] d W_{t}+\left[-i[H, \rho]+K \rho K-\frac{1}{2} K^{2} \rho-\frac{1}{2} \rho K^{2}\right] d t \\
& =-i[K, \rho] d W_{t}+\left[-i[H, \rho]-\frac{1}{2}[K,[K, \rho]]\right] d t
\end{aligned}
$$

while from Eqs. (3), (7b), and (11) we see that

$$
A=1-i K d W_{t}+\left(-i H-\frac{1}{2} K^{2}\right) d t=\exp \left[-i H d t-i K d W_{t}\right]
$$

Thus, in the case when the index set $N$ contains a single term, the transformation of Eq. (16) reduces to an infinitesimal stochastic unitary transformation.

\section{Acknowledgments}

This work was supported in part by the Department of Energy under Grant \#DEFG02-90ER40542. 


\section{References}

[1] See, e.g., V. Gorini, A. Frigerio, M. Verri, A. Kossakowski, and E. C. G. Sudarshan, Reports on Math. Phys. 13 (1978) 149.

[2] K. Kraus, Ann. Phys. (NY) 64 (1971) 311; E. B. Davies, Quantum Theory of Open Systems, Academic Press, London, 1976, Sec. 9.2.

[3] G. Lindblad, Commun. Math. Phys. 48 (1976) 119; V. Gorini, A. Kossakowski, and E.

C. G. Sudarshan, J. Math. Phys. 17 (1976) 821.

[4] A. Peres, quant-ph/9906023, Sec. V.

[5] K. R. Parthasarathy, An Introduction to Quantum Stochastic Calculus, Birkhäuser Verlag, Basel, 1992, Chapt. III.

[6] For an excellent exposition of the Itô calculus, see C. W. Gardiner, Handbook of Stochastic Methods, Springer-Verlag, Berlin, 1990, Chapt. 4. 